\title{
Vertical mode decomposition and model resolution
}

\author{
By PEDRO L. DA SILVA DIAS, Department of Meteorology, Instituto Astronômico e Geofísico, \\ Universidade de São Paulo,0100-São Paulo, Brazil and JOSÉ P. BONATTI, Department of \\ Meteorology, Instituto de Pesquisas Espaciais, Conselho de Desenvolvimento Cientifico e Tecnológico, \\ 12200-São José dos Campos, São Paulo, Brazil
}

(Manuscript received April 29; in final form July 26. 1985)

\begin{abstract}
The sensitivity of the vertical mode expansion to the location of the upper boundary of the model atmosphere and to the vertical resolution is studied. The vertical modes are determined using a $\sigma$-coordinate linearized primitive equation model with basic state at rest. The $\sigma$-levels are defined at standard pressure levels so as to avoid interpolation influence on the results. Two basic sets of experiments are performed: (a) varying the top pressure where $\sigma$ is zero while maintaining the vertical resolution in the interior unchanged; (b) fixing the top pressure and varying the vertical resolution. The results are analysed in terms of the energetics of the projection of gridded FGGE level III-b data at one particular grid point over tropical South America during the summer. It is found that the peak energy which occurs at an equivalent depth of the order of $220 \mathrm{~m}$ is approximately invariant with respect to experiments $a$ and $b$.
\end{abstract}

\section{Introduction}

The interpretation of the vertical atmospheric structure in terms of the vertical modes is analogous to the horizontal counterpart. In the recent literature, the horizontal projection has been applied to the global decomposition of $500 \mathrm{mb}$ data by Kasahara (1976), assuming the equivalent depth of the external mode, which is basically barotropic. More recent applications have been on the normal mode nonlinear initialization of modal decompositions (Daley, 1981). Since Rossby and gravity modes are basically different categories of motions, allowed by the dynamics of the primitive equation model of the atmosphere, it seems reasonable to explore the partition of energy between these modes. Further insight into the dispersive properties of the atmosphere can be gained by exploring the energy projection onto the various horizontal modes. However, the time scale of the horizontal modes is closely related to the vertical modes through the spectrum of equivalent depths of the stratified model. Thus, the projection onto horizontal modes is dependent on the choice of an equivalent depth and this involves the knowledge of the vertical energy partition among the vertical modes associated with the model.

Another point of view can be explored if the vertical structure is considered as an optimization problem in which maximization of variance by the minimum number of vertical modes is investigated: Holmström's (1963) analysis belongs to this category. Monin (1972) applied principal component analysis to this problem aiming at a compact representation of the vertical structure of the atmosphere in order to optimize the inversion techniques applicable to satellite temperature retrievals. It is interesting to note the similarity between the empirically determined vertical modes and those obtained as eigenfunctions of the vertical structure equation (Monin, 1972).

The vertical modes of the Australian Numerical Meteorological Research Center (ANMRC) primitive equation model (Bourke et al. 1977) formed the basis for the projection of global data by Kasahara \& Puri (1981). The vertical partition shows a peak at the external mode and a secondary peak at the $3 r d$ internal mode $(n=4)$. Puri (1983) discusses the nature of the modelled Hadley circulation by the ANMRC vertical modal space. 
It is shown that the moist convective parameterization is responsible for the 3rd internal mode dominance in the Hadley cell representation. Silva Dias \& Bonatti (1985) decomposed FGGE level III-b data over tropical South America onto the ANMRC vertical modes showing the dominance of the 3 rd internal mode in the energistics of the tropical circulations and the transition to the external mode dominance toward higher latitudes.

This paper has the purpose of investigating the sensitivity of vertical mode expansion to the location of the top pressure level and to the vertical resolution of the finite difference version of a $\sigma$-level primitive equation model based on the ANMRC version. The results are analysed in terms of the energetics of the projection of gridded FGGE level III- $b$ data at one particular grid point over tropical South America during the summer.

\section{Model equations}

The linearized primitive equations on the sphere, assuming a basic state at rest and with the vertical $\sigma$-coordinate, can be written as:

$$
\begin{aligned}
& \frac{\partial u^{\prime}}{\partial t}-2 \Omega \sin \phi v^{\prime}=\frac{1}{a \cos \phi} \frac{\partial P^{\prime}}{\partial \lambda}, \\
& \frac{\partial v^{\prime}}{\partial t}+2 \Omega \sin \phi u^{\prime}=-\frac{1}{a} \frac{\partial P^{\prime}}{\partial \phi}, \\
& \frac{\partial}{\partial t}\left[\frac{\partial}{\partial \sigma}\left(\frac{\sigma+\bar{q}_{\mathrm{t}}}{R \bar{\gamma}} \frac{\partial P^{\prime}}{\partial \sigma}\right)\right]-\nabla_{\sigma} \cdot V^{\prime}=0,
\end{aligned}
$$

with

$$
\sigma=\frac{P-P_{\mathrm{t}}}{P_{\mathrm{s}}-P_{\mathrm{t}}},
$$

where $P, P_{\mathrm{s}}$ and $P_{\mathrm{t}}$ are respectively local pressure, surface pressure and pressure at the top of the model atmosphere, and

$\bar{\gamma}=\frac{\kappa \bar{T}}{\sigma+\bar{q}_{\mathrm{t}}}-\frac{d \bar{T}}{\mathrm{~d} \sigma}$

is the static stability of the basic state at rest with temperature profile $\bar{T}(\sigma)$. The remaining symbols have the usual meteorological meaning except for

$$
P^{\prime}=g z^{\prime}+\frac{R \bar{T} \sigma q_{\mathrm{s}}^{\prime}}{\sigma+\bar{q}_{\mathrm{t}}}
$$

where

$\bar{q}_{\mathrm{t}}=\frac{P_{\mathrm{t}}}{\bar{P}_{\mathrm{s}}-P_{\mathrm{t}}}=\mathrm{constant}$

and

$q_{\mathrm{s}}^{\prime}=\ln \frac{P_{\mathrm{s}}-P_{\mathrm{t}}}{\bar{P}_{\mathrm{s}}-P_{\mathrm{t}}}$

with $\bar{P}_{\mathrm{s}}$ representing the average surface pressure of the basic state, $g$ is gravity, and $z^{\prime}$ is the perturbation geopotential height.

The above formulation is slightly different from Daley (1979) and Kasahara and Puri (1981) due to the inclusion of a top pressure level different from zero. However, eq (1) to (8) reduce to Kasahara and Puri's (1981) for $p_{\mathrm{t}}=0$.

Eq (1). (2) and (3) constitute a system of three equations in the three unknowns $u^{\prime}, v^{\prime}$, and $P^{\prime}$ which can be solved, provided the boundary conditions

$\frac{\partial P^{\prime}}{\partial \sigma}<\infty \quad$ at $\quad \sigma=0$.

$\frac{\partial P^{\prime}}{\partial \sigma}+\frac{\bar{\gamma}_{\mathrm{s}}}{\bar{T}_{\mathrm{s}}} P^{\prime}=0 \quad$ at $\quad \sigma=1$

are specified. The subscript "s" in (8) and (10) refers to the surface value of the indicated variable. The above boundary conditions are equivalent to the dynamical conditions $\dot{\sigma}=0$ at the top and bottom boundaries of the model.

The usual procedure to separate the vertical and horizontal structures associated with system (1), (2) and (3) is to consider the separation of variables

$\left[\begin{array}{l}u^{\prime} \\ v^{\prime} \\ P^{\prime}\end{array}\right](\lambda . \phi . \sigma, t)=\psi(\sigma)\left[\begin{array}{l}\hat{u} \\ \hat{v} \\ \hat{P}\end{array}\right](\lambda, \phi, t)$,

with vertical structure governed by

$\frac{\mathrm{d}}{\mathrm{d} \sigma}\left[\frac{\left(\sigma+\bar{q}_{\mathrm{t}}\right)}{R \bar{\gamma}} \frac{\mathrm{d} \psi}{\mathrm{d} \sigma}\right]+\frac{1}{D} \psi=0$, 
where $D$ is the separation constant. The boundary conditions associated with (12) are derived from (9) and (10) and can be expressed as

$$
\begin{aligned}
& \frac{\mathrm{d} \psi}{\mathrm{d} \sigma}=0 \quad \text { at } \quad \sigma=0, \\
& \frac{\mathrm{d} \psi}{\mathrm{d} \sigma}+\frac{\bar{\gamma}_{\mathrm{s}}}{T_{\mathrm{s}}} \psi=0 \quad \text { at } \quad \sigma=1 .
\end{aligned}
$$

The vertical structure equation (12) and the boundary conditions (13) and (14) define a Stürm-Liouville problem with $D_{n}(n=1,2,3 \ldots)$ as eigenvalues. The finite difference form of the vertical structure equation and the associated boundary conditions is in general not in the Stürm-Liouville form with orthogonal eigenfunctions. Orthogonal vertical modes simplify the representation of field variables and allows the study of energetics in each vertical mode separately (Kasahara and Shigehisa, 1983). Only certain forms or combinations of the finite differencing are in the Stürm-Liouville form. In Kasahara and Puri (1981), the vertical discretization of the vertical structure equation follows the staggering of the ANMRC model, which was also used by Silva Dias and Bonatti (1985) yielding the finite difference form of the Stürm-Liouville problem. In the present case, the same vertical discretization is used, except for the positioning of the vertical levels which are defined at the standard pressure levels.

A vertical sounding can be decomposed as a series of the eigenfunctions $\psi_{n, k}$ as

$$
\begin{aligned}
& {\left[\begin{array}{l}
u_{n} \\
v_{n} \\
p_{n}
\end{array}\right](\lambda, \phi)=\sum_{k=1}^{K} \psi_{n, k}} \\
& \times\left[\begin{array}{c}
u_{0}(\lambda, \phi, k) / \sqrt{g D_{n}} \\
v_{0}(\lambda, \phi, k) / \sqrt{g D_{n}} \\
{\left[z_{0}^{\prime}(\lambda, \phi, k)+\frac{R T(k) \sigma q_{\mathrm{s}, 0}^{\prime}(\lambda, \phi)}{g\left(\sigma+\bar{q}_{\mathrm{t}}\right)}\right] / D_{n}}
\end{array}\right],
\end{aligned}
$$

where $K$ represents the total number of vertical levels, $k$ is the particular level and the subscript "o" indicates the observed fields. The kinetic and available potential energies associated with each vertical mode at each grid point are defined by

$K_{n}=g \frac{D_{n}}{2}\left(u_{n}^{2}+v_{n}^{2}\right)$

$A_{n}=g \frac{D_{n}}{2} p_{n}^{2}$,

respectively. It is also useful to define the parameter $R_{n}$ which represents the \% of the total energy which is contained in the $n^{\prime}$ th mode as

$R_{n}=\frac{\left(K_{n}+A_{n}\right)}{\sum_{n=1}^{K}\left(K_{n}+A_{n}\right)} \times 100 \%$

\section{Vertical decomposition}

The geopotential height and horizontal velocity at standard pressure levels (up to $10 \mathrm{mb}$ ) and the surface pressure at $15^{\circ} \mathrm{S}$ and $56.25^{\circ} \mathrm{W}$ on February 2, 1979 at 00 GMT were subjected to the vertical decomposition described in Section 2. This

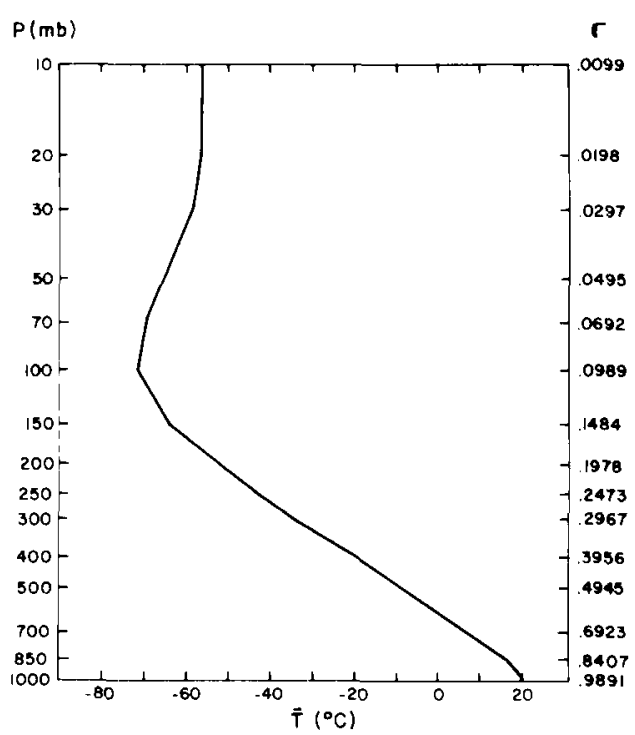

Fig. 1. Basic state temperature profile over the region limited by the grid points $\left(30^{\circ} \mathrm{N}, 135^{\circ} \mathrm{W}\right),\left(30^{\circ} \mathrm{N}\right.$, $\left.3.75^{\circ} \mathrm{W}\right),\left(45^{\circ} \mathrm{S}, 135^{\circ} \mathrm{W}\right)$ and $\left(45^{\circ} \mathrm{S}, 3.75^{\circ} \mathrm{W}\right)$ during the period January 29, 1979 to February 16, 1979. 
Table 1. Basic characteristics of the experiments

\begin{tabular}{lcc}
\hline Experiment & Top pressure $(\mathrm{mb})$ & Number of levels \\
\hline A1 & 0 & 15 \\
A2 & 30 & 12 \\
A3 & 70 & 10 \\
B & 0 & 8 \\
\hline
\end{tabular}

particular grid point of the FGGE data set was chosen on the basis of its representativeness of the perturbed tropical atmosphere which has been shown to be dominated by the 3rd internal mode $(n=4)$ of the ANMRC model, as discussed by Silva Dias and Bonatti (1985). The basic state (Fig. 1 ) is defined by the average temperature profile over the domain limited by the grid points $\left(30^{\circ} \mathrm{N}\right.$, $\left.135^{\circ} \mathrm{W}\right) .\left(30^{\circ} \mathrm{N}, 3.75^{\circ} \mathrm{W}\right),\left(45^{\circ} \mathrm{S}, 135^{\circ} \mathrm{W}\right)$ and $\left(45^{\circ} \mathrm{S}, 3.75^{\circ} \mathrm{W}\right)$, during the period 29 January 1979 to 16 February 1979, as in Silva Dias and Bonatti (1985). The $\sigma$-levels coincide with standard pressure levels. This choice eliminates possible interpolation effects from $p$-levels to $\sigma$-levels.

Several experiments were carried out by lowering the top pressure from $0 \mathrm{mb}$ to $70 \mathrm{mb}$ and reducing the vertical resolution by reducing standard pressure levels. Table 1 describes the basic characteristics of the experiments presented in this paper. Experiments A1, A2 and A3 allow the identification of the effects associated with lowering of the top pressure: Experiments B and A1 reveal the effect of vertical resolution.

\subsection{Vertical mode structure}

Figs. 2, 3, 4 and 5 show the vertical eigenfunctions for the allowed vertical eigenvalues $D_{n}$ and for each of the experiments defined in Table 1 , respectively. The vertical scale based on pressure in Figs. 2-5 is made the same for each of the experiments in order to emphasize the relationship among modes.

The vertical structure of the external mode $(n=1)$ in Experiments $\mathrm{A} 1, \mathrm{~A} 2$ and $\mathrm{A} 3$ shows little variation below $100 \mathrm{mb}$. This particular mode is characterized by a constant sign profile with a slight bulge in the lower troposphere. The lower vertical resolution experiment (B) also conserves the shape of the external mode below $100 \mathrm{mb}$. However, the equivalent depth decreases from 9822 $\mathrm{m}$ to $8709 \mathrm{~m}$ as the top level is lowered from $0 \mathrm{mb}$
Table 2. Equivalent height $(m)$ associated with experiments $A 1, A 2, A 3$ and $B$

\begin{tabular}{rrrrr}
\hline$n$ & A1 & A2 & A3 & \multicolumn{1}{c}{ B } \\
\hline 1 & 9822 & 9205 & 8709 & 9801 \\
2 & 3178 & 979 & 416 & 3149 \\
3 & 836 & 279 & 156 & 888 \\
4 & 385 & 145 & 71.2 & 435 \\
5 & 227 & 84.9 & 35.6 & 229 \\
6 & 145 & 62.2 & 24.9 & 96.3 \\
7 & 117 & 35.3 & 17.2 & 51.7 \\
8 & 80.0 & 24.8 & 12.6 & 38.8 \\
9 & 61.6 & 17.1 & 12.1 & \\
10 & 35.3 & 12.6 & 7.8 & \\
11 & 24.8 & 12.1 & & \\
12 & 17.1 & 7.7 & & \\
13 & 12.5 & & & \\
14 & 12.1 & & & \\
15 & 7.1 & & & \\
\hline
\end{tabular}

to $70 \mathrm{mb}$ (Table 2), implying a slight reduction of the time scale associated with the dispersive properties of the external mode. Lower vertical resolution has a negligible effect on the equivalent depth of the barotropic mode.

The lower order internal modes are compressed downwards as the top level is lowered from $0 \mathrm{mb}$ to $70 \mathrm{mb}$ as shown in Fig. 2; the number of zeroes in the vertical eigenfunctions is evidently the same, but the baroclinic character is accentuated. As indicated in Table 2, a clear relationship ceases to exist among equivalent heights for the low and intermediate order internal modes. Thus, comparison of the dispersive properties is complicated by the velocity scaling $\left(\sqrt{g D}_{n}\right)$ and the superposition of many vertical modes has to be considered. Contrary to the results of Experiments A, the lower vertical resolution (Experiment B) has little effect on the vertical profile of the eigenfunctions (Figs. 2 and 5) or on the equivalent depth of the lower order modes up to the $n=5$ mode (Table 2, columns A1 and B). The vertical structure is more influenced by the coarser vertical resolution at higher levels where the basic state temperature field shows more variation with height (Figs. 2-5).

The higher order modes associated with Experiments $\mathrm{A} 1, \mathrm{~A} 2$ and $\mathrm{A} 3$ show negligible dependence on the top pressure because their amplitude is confined to the lower troposphere (Figs. 2-4). Evidently the order of the mode varies $(n)$ because the number of permissible vertical modes is the 


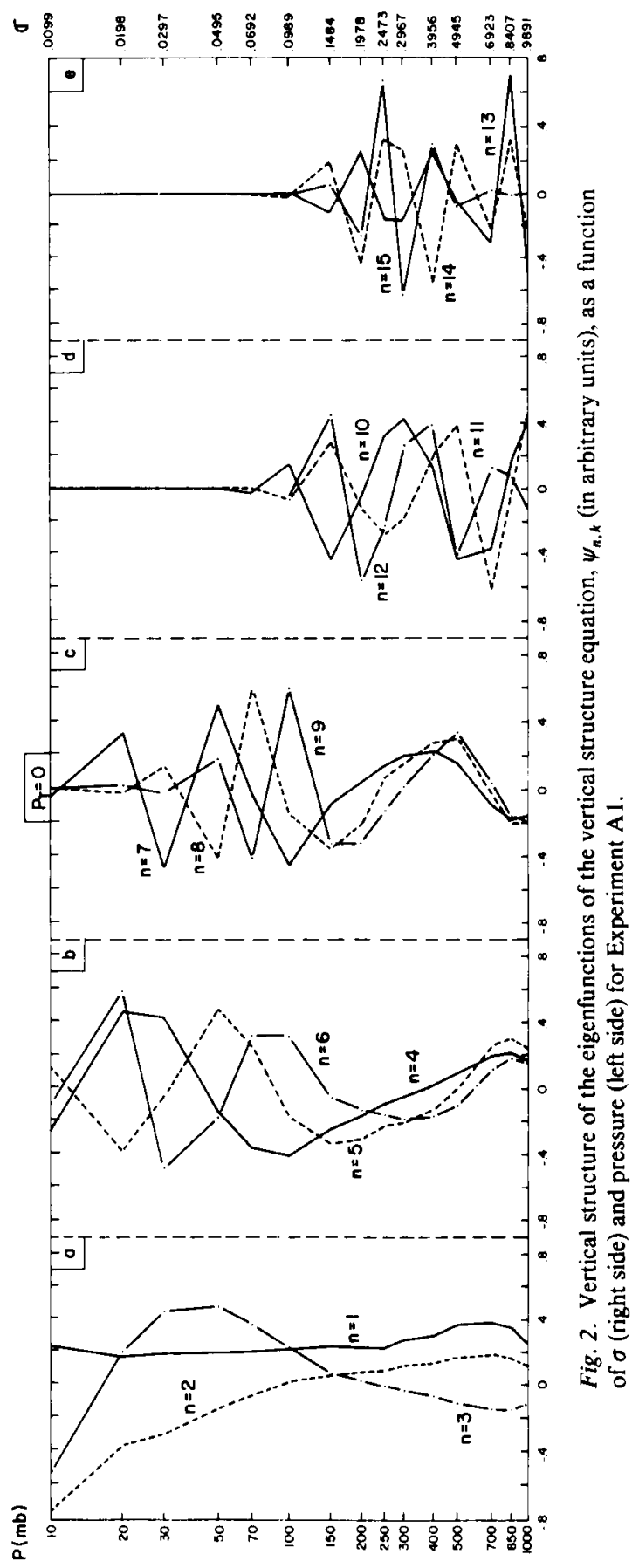

Tellus 38A (1986), 3 


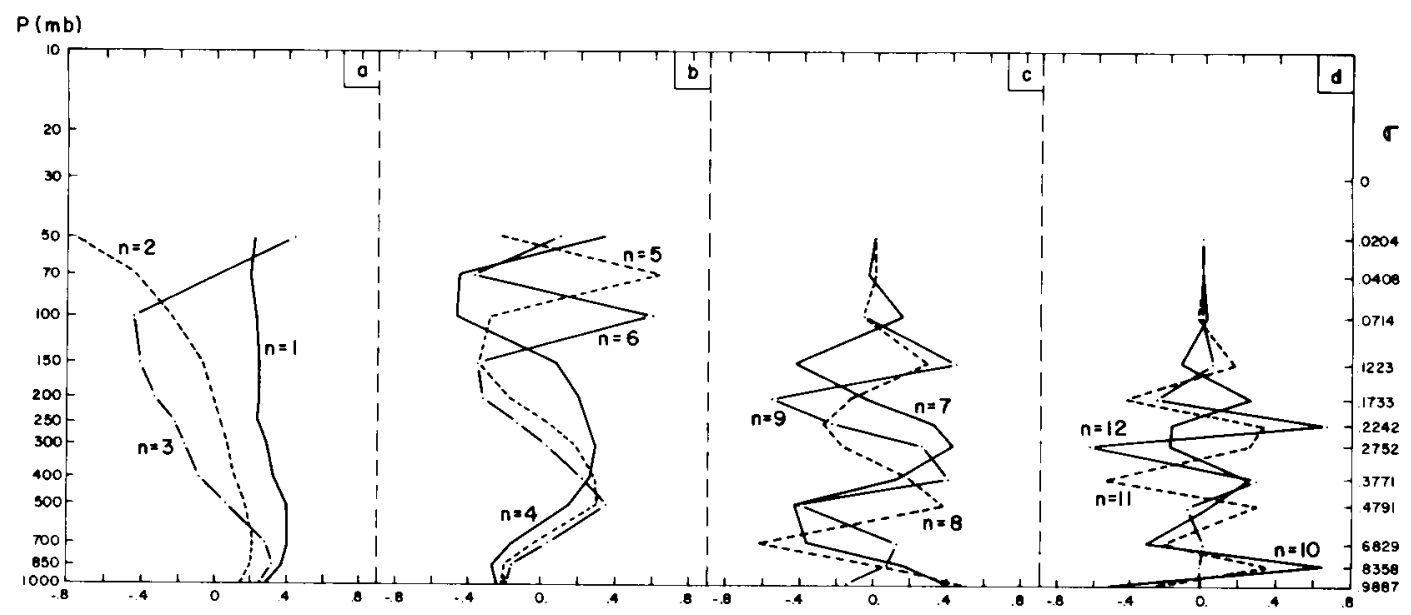

Fig. 3. Same as Fig. 2, but for Experiment A2.

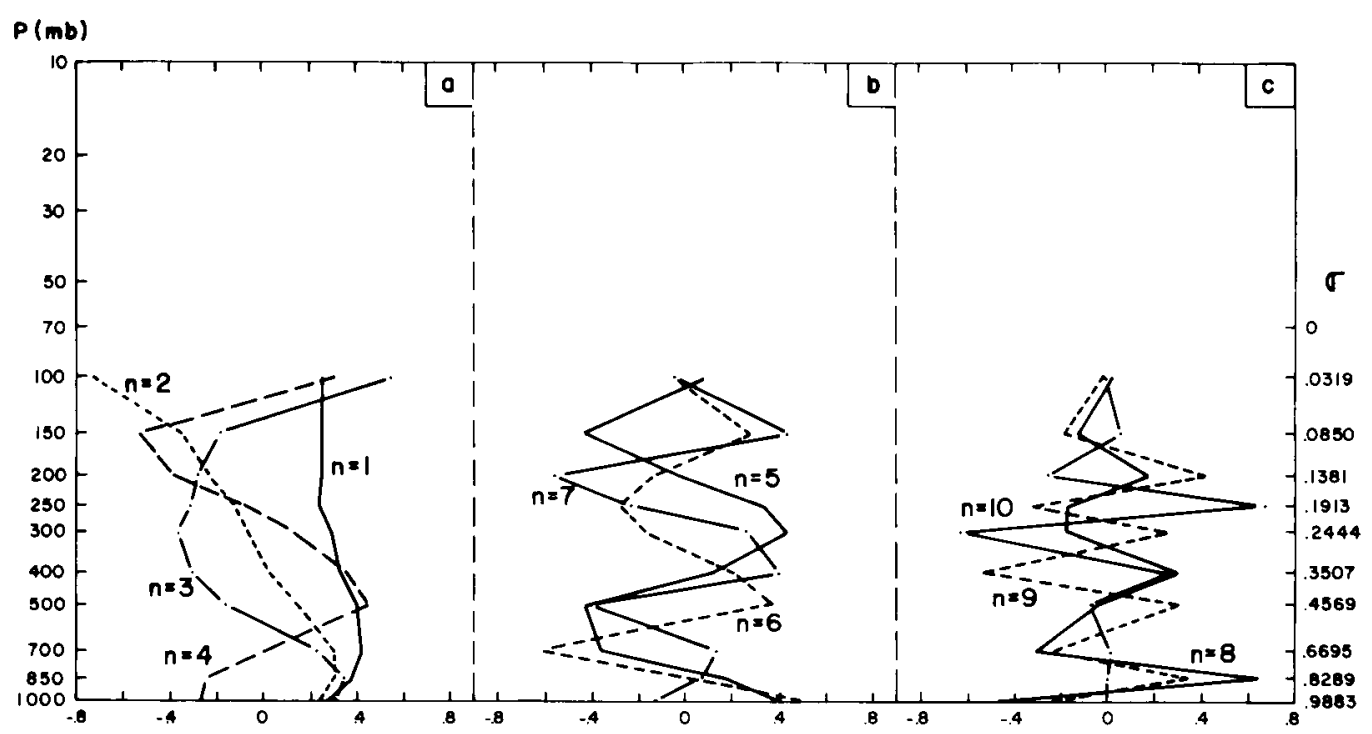

Fig. 4. Same as Fig. 2, but for Experiment A3.

number of vertical levels. Thus, the equivalent depth of the higher-order modes remains unchanged in Experiments A (Table 2) and, as a consequence, the lower tropospheric features, which are described by the higher order internal modes, are equally represented by the $\sigma$-level model described in Section 2, with top pressure at $0 \mathrm{mb}$ or $70 \mathrm{mb}$. Thus the dispersive properties of such lower trophospheric features are practically independent of the location of the top pressure. A comparison between the results of the Experiments Al and B indicates that, besides the exclusion of the detailed lower structure of the higher order modes in A, modes with similar equivalent depth also show discrepancies in their vertical structures. As an example, we can compare the 10th mode in Experiment A with the 8th mode of Experiment B, which have equivalent depths of $35.3 \mathrm{~m}$ and $38.8 \mathrm{~m}$, respectively (Table 2 ); although the general shape of the associated eigenfunctions is similar, the positioning of the zeroes differs by more than 100 mb (Figs. 2-5). 


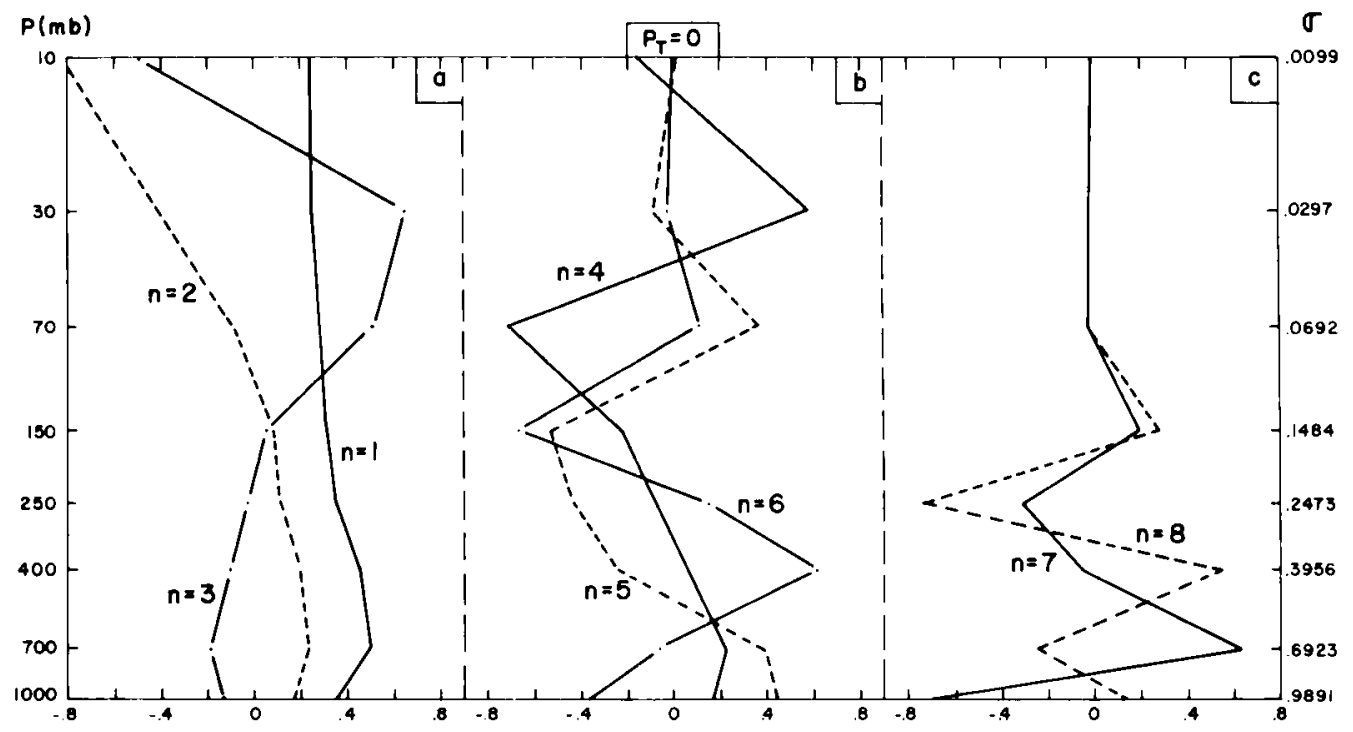

Fig. 5. Same as Fig. 2, but for Experiment B.

\subsection{Energetics of the vertical decomposition}

The \% of total energy $\left(R_{n}\right)$, the available potential energy $\left(A_{n}\right)$ and the kinetic energy $\left(K_{n}\right)$ as a function of the vertical mode $n$ and equivalent depth $D_{n}$ defined by (18), (17) and (16) are shown in Figs. $6 \mathrm{a}, 6 \mathrm{~b}$ and $6 \mathrm{c}$, respectively, for each of the experiment defined in Table 1. Fig. 6a clearly depicts the dominance of total energy within the range of $150 \mathrm{~m}$ to $250 \mathrm{~m}$ in the equivalent depth scale. The peak at approximately $220 \mathrm{~m}$ was also noticed by Silva Dias and Bonatti (1985) and its association with convective forcing was established by Puri (1983) and arrived at by Lim and Chang (1981, 1983), Gill (1980) and Silva Dias et al. (1983) from theoretical considerations. Fig. 6a shows that although the total energy changes as a function of the vertical mode index $n$, it is relatively invariant with respect to the equilvalent depth $D_{n}$. Lowering the top pressure to $70 \mathrm{mb}$ has the maximum impact, represented by the wider spectrum in Fig. 6a. Lower vertical resolution (comparing the results of Experiment Al with B) shows little effect for the external mode, as anticipated from the results of Section 3a, and a progressively larger influence for the internal modes.

When analysing the variation of available potential energy (Fig. 6b) and kinetic energy (Fig. 6c), a different picture emerges: the clear cut peak at the approximately $220 \mathrm{~m}$ equivalent depth mode is not present. In particular, the available potential energy shows a double-peaked structure at approximately $220 \mathrm{~m}$ and $30 \mathrm{~m}$, while the kinetic energy seems to show a gradual increase of magnitude as the equivalent depth increases (a factor of $10^{3}$ as $D_{n}$ varies from $10 \mathrm{~m}$ to $10^{4} \mathrm{~m}$ ). A comparison of Figs. $6 \mathrm{~b}$ and $6 \mathrm{c}$ also reveals that the kinetic energy is less than the available potential energy for small equivalent depth, the reverse being the case for larger equivalent depths.

The relationship between kinetic energy and available potential energy can be understood from a dispersion point of view, or equivalently from a group velocity argument. Large equivalent depth modes have higher group velocity and therefore the horizontal gravitational components are highly dispersive; this means that the adjustment of the mass and wind fields occurs in a rather short time scale towards a rotational state (Silva Dias and Schubert, 1979). However, the rotational modes have more kinetic energy than potential energy, except for the ultra-long waves (Longuet-Higgins, 1968; Kasahara, 1976; Bonatti et al., 1983), thus explaining the observed partition of energy. As the equivalent depth decreases, the dispersive characteristics are slowed down by the vertical scaling and a rotational constraint between the mass and wind fields is not enforced by the dynamics of the adjustment. This means that slowly dispersive

Tellus 38A (1986), 3 


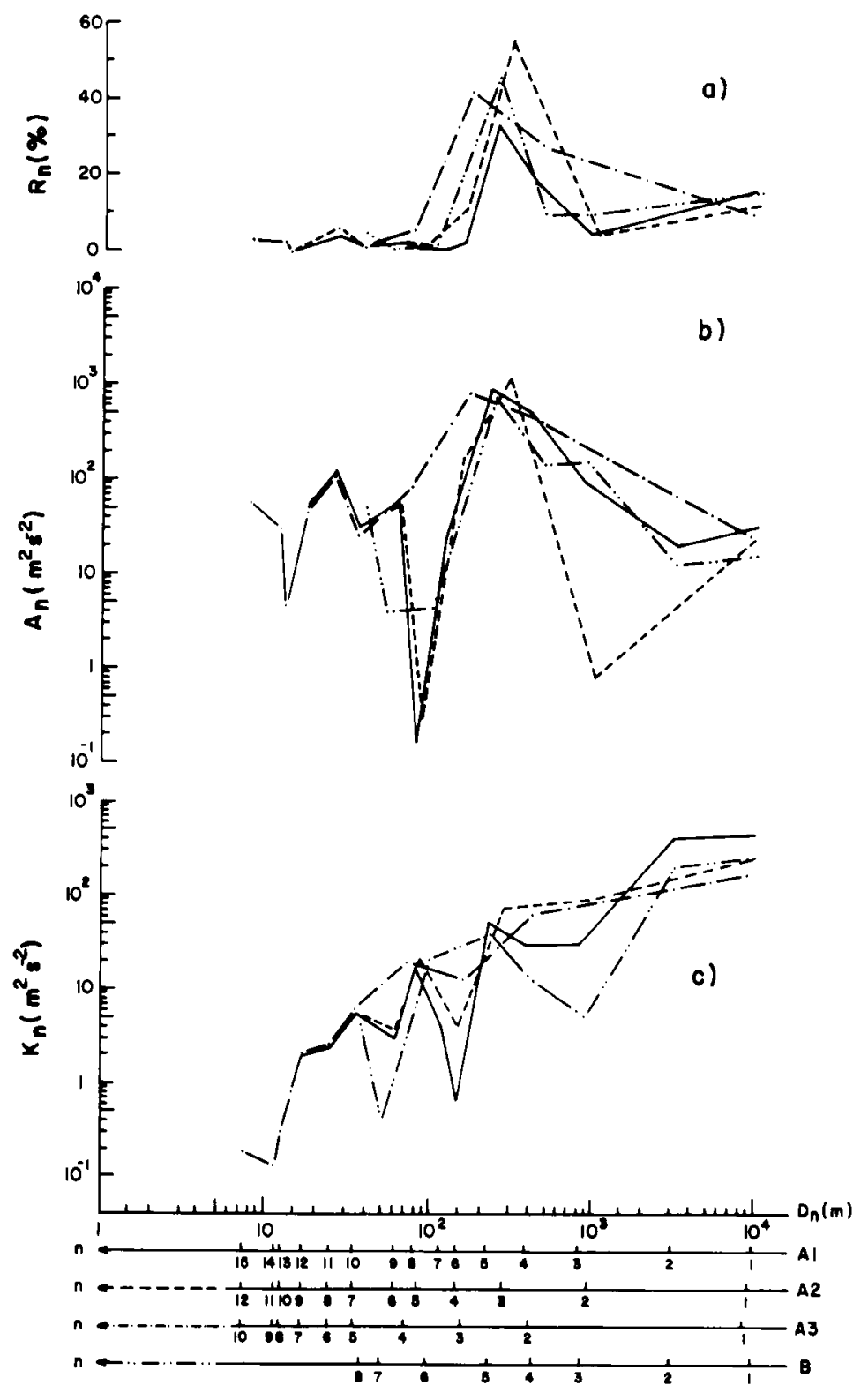

Fig. 6. \% of total energy $\left(R_{n}\right)$, available potential energy $\left(A_{n}\right)$ and kinetic energy $\left(K_{n}\right)\left(\mathrm{m}^{2} \mathrm{~s}^{-2}\right)$ as a function of equivalent depth $D_{n}(\mathrm{~m})$ and vertical mode index $n$ for Experiments A1, A2, A3 and B, as indicated.

gravity waves with small equivalent depth can have a locally important contribution to the observed fields. Because gravity-inertia waves have more potential energy than kinetic energy, there appears to be a larger component of geopotential contribution in the energetics.
Thus, from a dispersion argument it seems plausible to ascribe the dominance of the approximately $220 \mathrm{~m}$ mode in the total energy to available potential energy and not the kinetic energy. The secondary peak at approximately $30 \mathrm{~m}$ in $A_{n}$ (Fig. 6b) seems to be related to the boundary layer 
structure. The higher order internal modes were verified to have a large diurnal variation, reinforcing the low level character of these modes (not shown).

\section{Conclusion}

In the previous sections, we have discussed the sensitivity of the vertical modes of the linearized primitive equations $\sigma$-coordinate to the location of the top level and to vertical resolution. We examined the energetics of the FGGE level III-b vertical fields at a particular grid point over tropical South America during summer. It is found that the total energy peaks at approximately $220 \mathrm{~m}$ equivalent depth mode, which is practically invariant with respect to change in the location of the top level pressure (lowered from $0 \mathrm{mb}$ to $70 \mathrm{mb}$ ) and to the vertical resolution (halving the number of levels). The total energy peak is also seen to be related to the distribution of available potential energy with kinetic energy showing a gradual increase as the equivalent height is increased. These results give further observational support to the modelling work of Gill (1980), Lim and Chang (1981), Silva Dias et al. (1983).

The energy content as a function of vertical mode index is clearly a function of latitude as shown in Silva Dias and Bonatti (1985); at higher latitudes more energy is contained in the external mode. The results presented in this paper concerning the vertical structure of the eigenmodes for the series of experiments, allow us to conclude that vertical mode energetics of Silva Dias and Bonatti (1985) are representative.

It is also interesting to compare the vertical modes shown in Fig. 2 of Silva Dias and Bonatti (1985), which were obtained at different interpolated pressure levels ( 9 modes), with the eigenmodes of experiments A1 and B ( 15 and 8 modes, respectively). Modes with similar equivalent depth show similar vertical structure, and the higher order internal modes are better described with the interpolated scheme than with the course resolution version (Experiment B). This last result is a consequence of the improved vertical resolution in the lower troposphere in Silva Dias and Bonatti (1985).

The dependence of observed energetics on the equivalent depth can be interpreted as a result of the adjustment of the mass and wind fields and the energetics of rotational and gravitational modes. One question that can be raised at this point is related to the difference between observed vertical structures and objectively analysed structures. The latter are influenced by the model dynamics through the initial guess field, and in order to answer the question, further work has to be done, comparing, for instance, FGGE level II-b with FGGE level III-b vertical decompositions.

It is important to notice that although the horizontal dispersive properties do not seem to be greatly affected by the location of the top level or vertical resolution for the predominant vertical modes, nothing has been discussed concerning the vertical dispersion. The top lid, evidently, has a reflective character (Lindzen et al. 1970) and lowering the top restricts the vertical dispersion. This point has been stressed by Geisler and Stevens (1982) concerning Gill's (1980) work, regarding the response of the stationary tropical atmosphere to localized heat sources. This is another important aspect that can be studied with vertical modal projection.

\section{Acknowledgments}

The authors acknowledge helpful discussions with Wayne Schubert, Akira Kasahara, Antonio D. Moura and V. B. Rao. The figures were drafted by INPE Graphics Department and the typing was performed by Sueli A. F. V. Camargo Pinto.

One of the authors (P. L. da Silva Dias) has benefited from a CNPq fellowship and a FINEP/ USP/IAG grant.

\section{REFERENCES}

Bonatti, J. P., Silva Dias, P. L. and Moura, A. D. 1983. Hough functions: theory and utilization. INPE Report no. INPE-2697-RPE/429, 271 pp. In Portuguese. (Available from Instituto de Pesquisas Espaciais,
INPE, CP 515, 12200. São José dos Campos, SP, Brazil.)

Bourke, W., McAvaney, B., Puri, K. and Thurling, R. 1977. Global modeling of atmospheric flow by 
spectral methods. Methods Comput. Phys. 17, 268324.

Daley, R. 1979. The application of nonlinear normal mode initialization to an operational forecast model. Atmos. Ocean 17, 97-124.

Daley, R. 1981. Normal mode initialization. Rev. Geophys. Space Phys. 19, 450-468.

Geisler, J. E. and Stevens. D. E. 1982. On the vertical structure of damped steady circulation in the tropics. Q.J. R. Meteorol. Soc. 108, 57-93.

Gill, A. E. 1980. Some simple solutions for heat induced tropical circulation. Q.J.R. Meteorol. Soc. 106, 447-462.

Holmström, I. 1963. On a method for parametric representation of the state of the atmosphere. Tellus 15 . 127-149.

Kasahara. A. 1976. Normal modes of ultralong waves in the atmosphere. Mon. Wea. Rev. 104, 669-690.

Kasahara, A. and Puri, K. 1981. Spectral representation of three-dimensional global data by expansion in normal mode functions. Mon. Wea. Rev. 109, 37-51.

Kasahara, A. and Shigehisa, Y. 1983. Orthogonal vertical normal modes of a vertically staggered discretized atmospheric model. Mon. Wea. Rev. 111, 1724-1735.

Lim, H. and Chang, C.-P. 1981. A theory of midlatitude forcing of tropical motions during winter monsoons. $J$. Atmos. Sci. 38, 2377-2392.
Lim, H. and Chang, C.-P. 1983. Dynamics of teleconnexions and Walker circulations forced by equatorial heating. J. Atmos. Sci. 40, 1897-1915.

Lindzen, R., Batten, E. and Kim, J. 1970. Oscillations in atmospheres with tops. Mon. Wea. Rev. 96, 133-140.

Longuet-Higgins, M. S. 1968. The eigenfuctions Laplace's tidal equations over a sphere. Phil. Trans. Soc. 262A, 511-607.

Monin, A. S. 1972. Weather forecasting as a problem in physics. MIT Press, 199 pp.

Puri, K. 1983. The relationship between convective adjustment, Hadley circulation and normal modes of the ANMRC spectral model. Mon. Wea. Rev. III, 23-33.

Silva Dias, P. L. and Bonatti, J. P. 1985. A preliminary study of the observed vertical mode structure of the summer circulation over tropical South America. Tellus 37A, 185-195.

Silva Dias, P. L. and W. H. Schubert, 1979. The dynamics of equatorial mass-fow adjustment. Atmospheric Science Paper no. 312. Department of Atmospheric Science, Colorado State University, Fort Collins, Colorado 80523, 203 pp.

Silva Dias, P. L., Schubert, W. H. and DeMaria, M. 1983. Large-scale response of the tropical atmosphere to transient convection. J. Almos. Sci. 40, 2689-2707. 